\title{
What basal membranes can tell us about viscous forces in
}

\section{Drosophila ventral furrow formation}

Amanda Nicole Goldner ${ }^{1}$ and Konstantin Doubrovinski ${ }^{1,2, *}$

${ }^{1}$ Green Center for Systems Biology, Department of Bioinformatics, University of Texas

Southwestern Medical Center, 5323 Harry Hines Blvd., Dallas, TX, USA 75390

${ }^{2}$ Lead Contact

*Correspondence: konstantin.doubrovinski@utsouthwestern.edu 


\section{SUMMARY}

Ventral furrow (VF) formation in Drosophila melanogaster is an important model of epithelial folding. Past studies of VF formation focus on apical forces in driving folding, while other forces are largely unexplored. When basal membrane formation is genetically blocked using anillin knockdown (scra RNAi), the VF can still fold. To interpret our observations, we developed a computational model of VF formation that explicitly simulates the flows of viscous cytoplasm. The viscosity included in our model is required for tissue invagination in the absence of basal membranes. This strongly suggests that epithelial folding requires the presence of viscous shear forces from cytoplasm. Our model characterizes folding during VF formation as a "swimming phenomenon", where tissue deforms by pushing against the ambient viscous surroundings. Since VF formation is successful in scra RNAi embryos, we propose that models of gastrulation should be able to replicate this folding in the absence of basal membranes.

KEYWORDS: gastrulation, morphogenesis, anillin, epithelium, Drosophila, forces, viscosity, basal 


\section{INTRODUCTION}

Epithelial folding occurs throughout many stages in animal development (Leptin, 1991; Martinez Arias \& Stewart, 2003), including neurulation (Maruyama \& Andrew, 2012) and lung bud formation (Kim et al., 2013). During Drosophila gastrulation, the initially-monolayered embryo becomes multilayered through epithelial folding (Campos-Ortega \& Hartenstein, 1985; Kohler, 1994).

After fertilization of a Drosophila egg, the nuclei undergo 13 division cycles and migrate to the periphery of the embryo. Since nuclear division at this stage is not accompanied by cytokinesis, this results in an embryo that is essentially a large multinucleate cell encased in a rigid vitelline membrane (Leptin, 1991; Zalokar \& Erk, 1976). Subsequently, lateral membranes grow inwards to compartmentalize peripheral nuclei into individual cells, forming the epithelium in a process known as cellularization (Campos-Ortega \& Hartenstein, 1985; Lecuit \& Wieschaus, 2000). Throughout the course of cellularization, cells remain open to the yolk sac such that there is a "hole" on the basal side of each cell (see schematic in Figure S1) (Krueger et al., 2019; Loncar \& Singer, 1995; Mazumdar \& Mazumdar, 2002).

As cellularization completes, gastrulation begins with the folding of the ventral furrow (VF) (Campos-Ortega \& Hartenstein, 1985; Leptin \& Grunewald, 1990; Sweeton et al., 1991; Turner \& Mahowald, 1977). VF formation involves a sequence of shape changes in a subset of ventral cells, starting with constriction of their apical membranes (Kam et al., 1991; Leptin, 1991). As VF formation proceeds, lateral membranes lengthen before eventually shortening again as the basal membranes seal and the ventral tissue invaginates to form a furrow (Lye \& Sanson, 2011; Sweeton et al., 1991; Turner \& Mahowald, 1977). These shape changes are driven by actomyosin contractility in the membrane-associated cytoskeleton (Martin et al., 2009, 2010). Since 
cellularization is still ongoing during the initial apical constriction phase of VF formation, cells remain open and only start sealing basally as tissue invagination begins. The simultaneous occurrences of basal membrane formation and epithelial folding indicate one process may rely on the other. Past studies of VF formation focus on the role of apical constriction in driving epithelial folding (Sawyer et al., 2010). However, the relative contributions of forces in the various cellular domains remain elusive, and the contribution of basal membranes to VF formation is largely unexplored.

\section{RESULTS AND DISCUSSION}

\section{Basal membranes are not required for VF formation}

We began by exploring the role of basal membranes in VF formation. Loss-of-function mutations in Anillin (Scraps) - a component of the contractile ring scaffold that narrows to seal off cells and form basal membranes - delay basal membrane formation (Field et al., 2005; Thomas \& Wieschaus, 2004; Xue \& Sokac, 2016). However, gastrulation has not been characterized in this genetic background. For experimental simplicity, we first replicated this phenotype via maternal GAL4-driven expression of short hairpin RNA against scra (UASscraTRiP). Live imaging of embryos expressing maternal GAL4, UAS-scraTRiP, and a membrane marker (UAS-Nrt-GFP) showed that the effects of scra RNAi during cellularization were indistinguishable from those seen in Anillin loss-of-function mutants (Figure S2) (Field et al., 2005; Thomas \& Wieschaus, 2004; Xue \& Sokac, 2016). To better characterize gastrulation in this background, we prepared sections of heat-fixed gastrulas stained for Neurotactin, Snail, and DAPI (membrane, mesodermal, and nuclear markers, respectively), see Figure 1. As a control, embryos of similar genetic background without UAS-scraTRiP were 
stained according to the same protocol (Figures 1A-C). Basal membranes never form in scra RNAi gastrulas, yet the VF is remarkably still capable of folding to its usual depth (Figures 1GI).

\section{Membrane lengths are altered in scra RNAi embryos}

We were interested to see if there were any morphological differences between scra RNAi and control embryos. To this end, we used bar charts to compare the distribution of lateral membrane lengths along transverse sections of scra RNAi (Figures 1D-F) and control (Figures 1J-L) embryos at various stages of VF formation. In control embryos, distributions exhibit a curve with two peaks along the mesoderm: peripheral cells are shortest, followed by the most central cells, while cells at the boundary between these two regions are longest (Figures 1D-F). As apical constriction proceeds, lateral membranes lengthen (Figures 1D-E), then shorten throughout invagination as the VF forms more fully (Figures 1E-F). While the temporal lengthen-thenshorten pattern of morphological changes remains relatively unaffected in scra RNAi embryos (Figure S3), the spatial distributions of membrane lengths along the anillin depleted-VF have only one peak (Figures 1J-L). This difference arises in the degree to which various regions of the VF lengthen and shorten. Between apical constriction and invagination, central VF cells in scra RNAi embryos lengthen more than those of control embryos (Figure S3B). Over the same period, peripheral VF cells in scra RNAi embryos shorten less than those of control embryos (Figure S3C). 
We also measured the length of ectodermal membranes (outside the VF) at the same three stages of VF formation (Figure S4). In both cases, ectodermal membranes lengthen during VF formation. However, ectodermal lengthening is delayed in scra RNAi embryos (Figure S4).

\section{scra RNAi VFs remain folded despite degradation of lateral membranes}

Anillin mutants additionally present with membrane degradation and nuclear displacement (Field et al., 2005). Depletion of anillin in scra RNAi gastrulas also results in the gradual disintegration of lateral membranes, which eventually form vesicles (Figure S5). Membrane degradation increases in severity over time. Some VF cells become multinucleated, and VF nuclei are intermittently displaced into the yolk sac (Figures 1G-I). These findings suggest that Anillin plays a role in VF formation - not only during actin ring closure and basal membrane formation, but also in maintaining membrane stability.

The possibility remained that the phenotypes seen in immunostainings reflected differences in Neurotactin localization and not the presence or absence of membranes. To substantiate our findings, we examined the fine structure of epithelial membranes using transmission electron microscopy (TEM). Specifically, we froze gastrulas of various stages under high pressure, subjected them to freeze substitution, made 60-70nm transverse cross sections, and imaged them under TEM. At early stages of VF formation, interstitial spaces between ventral cells are visible in control embryos at low magnification (Figure 2A) indicating the presence of intact membranes (traced in green). In early stage scra RNAi gastrulas, these interstitial spaces are less intact, and lateral membranes have started disintegrating into vesicles (traced in red) (Figure 2B). Ventral cells in late stage control gastrulas have intact lateral and basal membranes (Figure 2C), all of which are absent in late stage scra RNAi gastrulas. Specifically, at the late stage, interstitial spaces are no longer visible and have been replaced by an increased number of vesicles (Figure 
2E). These TEM results confirm that the VF is indeed capable of forming in scra RNAi embryos, and the furrow retains its shape in the absence of basal, and subsequently, lateral membranes.

\section{VF formation without basal membranes can be explained by viscosity}

Many existing models of VF formation require volume conservation within cells (Rauzi et al., 2013). It is unclear how such models could describe a mutant in which basal membranes are missing. A mechanistic question remains: how are scra RNAi embryos capable of forming a furrow while cells remain open? To explore this question, we developed a series of computational models of gastrulation, in which a set of initially rectangular model "cells" represents a transverse cross-section of the embryonic epithelium. In these models, each cell is exclusively elastic along its surface. Dynamics are driven by contractile stresses in the different membranes (note that when we refer to membranes in this section, we imply the membraneassociated cytoskeleton instead of the lipid bilayer). Specifically, we assume that all membranes are subjected to a relatively small amount of constitutive contractile "pre-stress". The 16 mesodermal cells in our model have additional stress in their apical and lateral membranes. Technical details of model implementation are described in Supplemental Methods; the code used to generate the results is publicly available under https://github.com/doubrovinskilab/anillin_code.

In the most minimal version of this model, the mesoderm constricts apically and then invaginates (Figures 3A-C). When this model is modified so that cells exchange their internal contents freely with the yolk sac, (in other words basal membranes are absent to mimic scra RNAi embryos), the furrow does not form although apical constriction does occur (Figures 3D-F). We then modified this model to include a viscous cellular interior. Strikingly, this modification resulted in complete mesoderm invagination in the absence of basal membranes, mimicking our 
observations in scra RNAi embryos (Figures 3G-I). Importantly, the assumption of a highly viscous interior is based on physical measurements of embryonic Drosophila epithelium (Doubrovinski et al., 2017). Furthermore, a model which includes both viscosity and basal membranes (Figures 3J-L) results in very similar dynamics to the model with basal membranes but no viscosity (Figures 3A-C).

Finally, we asked if our model could reproduce consistent differences in the distribution of membrane lengths between scra RNAi and control. Beginning mid-way through gastrulation in control embryos, ventral cells that immediately flank the center of the mesoderm are the longest (Figure 1E). Our model including viscosity indeed reproduces this observation (compare Figures 3I and 3L). Remarkably however, this result was not reproduced during late VF formation with the parameter set we initially chose (Figure S6C), since the longest membranes in the control model were found in the middle. However, our in vivo observations were readily replicated in silico by simply increasing the magnitude of active stress in the basal domain of all cells; these are the results shown in Figure 3. Specifically, our model qualitatively replicates in vivo differences in "outer" membranes (edge of VF) vs. center membrane (middle of VF) length during late VF formation under control and scra RNAi conditions. For control conditions both in vivo and in silico, outer membranes are $15.8 \%$ longer than center VF membranes. Under scra RNAi conditions, outer membranes are $12.7 \%$ shorter in vivo and $17.7 \%$ shorter in silico (Figure S7). This indicates that the magnitude of active stresses on the basal surface can control the fine details of furrow shape, despite not being required for invagination. Our computational result can also be understood intuitively: when a curved surface is subject to in-plane stress, it tends to flatten. This effect is strongest where curvature is greatest. In our case, the curvature of the basal 
side is greatest precisely where mesoderm is flanked by the ectoderm. In this way, those flanking cells become significantly longer along the lateral direction if basal stress is increased.

To summarize, our minimal model accounts for both the invagination of the mesoderm in the absence of basal membranes as well as for the qualitative differences in mesodermal thickness between scra RNAi and control gastrulas. The key feature of our model is accounting for the viscous drag (i.e. shear) on the membranes from the ambient cytoplasm. In the absence of this effect, no invagination will result in the case when cells are not sealed basally. Basal membranes help confine the cytoplasm to the cellular interior, but viscous shear forces can have a similar effect. If viscosity is high enough, shear forces will prevent any appreciable amount of cytoplasm from escaping through the basal opening before invagination completes. Since invagination is largely unperturbed in the absence of basal membranes, our modeling results strongly suggest that viscous shear is a key physical effect involved in driving tissue folding.

\section{METHODS}

\section{Drosophila genetics}

For anillin depletion experiments (Figures 1-2), we used the following genotype: UAS-Nrt-eGFP; P\{y[+t7.7] $\mathrm{v}[+\mathrm{t} 1.8]=$ TRiP.GL01269 $\}$ attP2/mat15-GAL4. TRiP.GL01269 was derived from RRID:BDSC 41841 (Bloomington Drosophila Stock Center); mat15-GAL4 was derived from RRID:BDS_80361 (Bloomington Drosophila Stock Center). To generate UAS-Nrt-eGFP, full-length Nrt-RB was cloned by PCR from LD22004 (Drosophila Genomics Resource Center stock \#5736) and inserted into pPWG (Drosophila Genomics Resource Center stock \#1078). This plasmid was injected by BestGene using P-element insertion. Embryos were collected from female progeny on grape agar plates supplemented with yeast paste after $\geq 3.5 \mathrm{hrs}$.

\section{Fluorescent immunohistochemistry}

Fly embryos were heat-methanol fixed as described previously (Müller \& Wieschaus, 1996). The block, primary antibody, and secondary antibody steps were all done overnight with nutation at $4^{\circ} \mathrm{C}$. Primary and secondary antibodies were diluted in block solution (1x PBS, $0.1 \%$ Triton X-100, 5\% heat shocked goat serum or 0.2\% BSA). 
Antibodies used include mouse anti-Neurotactin (1:50) (DSHB), guinea pig anti-Snail (1:2000) (gift from Eric Wieschaus), goat anti-mouse IgG-Alexa Fluor 488 (1:500) (Invitrogen), and goat anti-guinea pig IgG-Alexa Fluor 568 (1:500) (Invitrogen). Nuclear staining was done using DAPI (1 $\mu \mathrm{g} / \mathrm{mL})$ (Invitrogen).

\section{Confocal fluorescence imaging of embryo sections}

Immunostained fly embryos were staged in 1x PBS with $0.1 \%$ Triton X-100 under bright field on an Accu-Scope dissection scope. A coverslip was prepared by cutting off $\sim 1 \mathrm{~cm}$ of the short edge and placing it in the center of the coverslip, perpendicular to the long edge, then adding a linear pool of AquaPolymount along the supporting glass strip. Selected gastrulas were transferred to pool of mounting medium and sectioned along the dorsal-ventral axis using a 22g needle. Embryo halves were positioned cut side down, leaning against the supporting glass strip. Zstacks were imaged using a Plan-Apochromat 63x/1.40 Oil objective on a Zeiss LSM 700 confocal microscope. For immunohistochemistry experiments, we fixed and stained samples over the course of several weeks, until we had at least 40 separate gastrulas imaged. From these, we selected all samples in which the morphology had not been significantly distorted by sectioning damage or angle - these results are collected in Figure 1.

\section{Statistical analysis of immunostaining results}

For the purposes of all experiments, all samples are biological replicates. We binned data from bar charts in Figure 1 (19 positions, -9 to 9) in sequential groups of 3, producing a plot with 6 average values of membrane lengths along the VF. We then compared experimental groups pairwise using Fisher's linear discriminant analysis. Specifically, we produced two sets of projections on the direction normal to the discriminant hyperplane, each set corresponding to one of the experimental groups being compared. Finally, the significance of the difference between the two sets of projections was assayed using the pairwise Kolmogorov-Smirnov test. An exception was made in the case of late VF formation scra RNAi embryos, where we only binned data from 15 positions (-7 to 7) - this was due to a smaller sample size for this group. For Figure S4, all data were used in analysis.

\section{Transmission electron microscopy}

A combined high pressure freezing and freeze substitution (HPF/FS) method was used to fix fly embryos as described elsewhere (Zhang \& Chen, 2008). Briefly, a Wohlwend Compact 03 high pressure freezer was used to fix the embryos. Samples were freeze substituted in $1 \%$ osmium tetroxide, $0.1 \%$ uranyl acetate in $98 \%$ acetone and $1 \%$ methanol using a Leica EM AFS2. The embryos were embedded in Embed-812 resin and polymerized in a $60^{\circ} \mathrm{C}$ oven overnight. Blocks were sectioned with a diamond knife (Diatome) on a Leica Ultracut 7 ultramicrotome (Leica 
Microsystems) and collected onto slot grids and post stained with $2 \%$ aqueous uranyl acetate and lead citrate. Images were acquired on a JEOL JEM-1400 Plus TEM equipped with a LaB 6 source operated at $120 \mathrm{kV}$ using an AMT-BioSprint 16M CCD camera. For TEM experiments, we continuously staged and fixed embryos over the course of 6-9 hours. Embryo sections shown in Figure 2 are representative of 5 (Figure 2A), 3 (Figure 2B), 11 (Figure 2C), or 4 (Figure 2D) samples. From these, we selected all samples in which the morphology had not been significantly distorted by ice/mechanical damage or other staining artifacts.

\section{Mathematical modeling}

For a detailed description, please see the Supplemental Materials.

\section{AUTHOR CONTRIBUTIONS}

Conceptualization, A.G. and K.D.; Supervision, K.D., Methodology, A.G. and K.D., Software, K.D., Investigation, A.G. and K.D., Data Curation, A.G. and K.D., Formal Analysis, A.G. and K.D., Visualization, A.G., Writing - original draft, A.G., Writing, review and editing, A.G. and K.D., Funding Acquisition, K.D.

\section{ACKNOWLEDGEMENTS}

We would like to thank Miriam Osterfield (UT Southwestern Medical Center) for technical advice, experimental discussions, and manuscript comments. This work was supported by the Welch Foundation award I-1950-20180324 and the National Institutes of Health grant 1R01GM134207-01. Stocks obtained from the Bloomington Drosophila Stock Center (NIH P40OD018537) were used in this study. The authors would like to acknowledge the assistance of the UT Southwestern Electron Microscopy Core, especially Anza Darehshouri. The NIH Shared Instrumentation award 1S10OD021685-01A1 to Katherine Luby-Phelps allowed for use of the JEOL JEM-1400 Plus TEM. 


\section{COMPETING INTERESTS}

The authors declare no competing interests.

\section{REFERENCES}

Campos-Ortega, J. A., \& Hartenstein, V. (1985). The Embryonic Development of Drosophila melanogaster (1st ed.). Springer. https://doi.org/https://doi.org/10.1007/978-3-662-02454-6

Doubrovinski, K., Swan, M., Polyakov, O., \& Wieschaus, E. F. (2017). Measurement of cortical elasticity in Drosophila melanogaster embryos using ferrofluids. Proceedings of the National Academy of Sciences, 114(5), 1051-1056. https://doi.org/10.1073/pnas.1616659114

Field, C. M., Coughlin, M., Doberstein, S., Marty, T., \& Sullivan, W. (2005). Characterization of anillin mutants reveals essential roles in septin localization and plasma membrane integrity. Development, 132(12), 28492860. https://doi.org/10.1242/dev.01843

Kam, Z., Minden, J. S., Agard, D. A., Sedat, J. W., \& Leptin, M. (1991). Drosophila gastrulation: analysis of cell shape changes in living embryos by three-dimensional fluorescence microscopy. Development, 112(2), 365370. http://dev.biologists.org/content/112/2/365.abstract

Kim, H. Y., Varner, V. D., \& Nelson, C. M. (2013). Apical constriction initiates new bud formation during monopodial branching of the embryonic chicken lung. Development, 140(15), 3146 LP - 3155. https://doi.org/10.1242/dev.093682

Kohler, R. E. (1994). Lords of the Fly: Drosophila Genetics and the Experimental Life (1st ed.). University of Chicago Press.

Krueger, D., Quinkler, T., Mortensen, S. A., Sachse, C., \& De Renzis, S. (2019). Cross-linker-mediated regulation of actin network organization controls tissue morphogenesis. Journal of Cell Biology, 218(8), 2743-2761. https://doi.org/10.1083/jcb.201811127

Lecuit, T., \& Wieschaus, E. (2000). Polarized Insertion of New Membrane from a Cytoplasmic Reservoir during Cleavage of the Drosophila Embryo. Journal of Cell Biology, 150(4), 849-860. 
https://doi.org/10.1083/jcb.150.4.849

Leptin, M. (1991). Mechanics and Genetics of Cell Shape Changes during Drosophila Ventral Furrow Formation. In R. Keller, W. H. Clark Jr., \& F. Griffin (Eds.), Gastrulation: Movements, Patterns, and Molecules (1st ed., pp. 199-211). Springer. https://doi.org/https://doi.org/10.1007/978-1-4684-6027-8

Leptin, M., \& Grunewald, B. (1990). Cell shape changes during gastrulation in Drosophila. Development, 110(1), 73-84. http://dev.biologists.org/content/110/1/73.abstract

Loncar, D., \& Singer, S. J. (1995). Cell membrane formation during the cellularization of the syncytial blastoderm of Drosophila. Proceedings of the National Academy of Sciences, 92(6), 2199-2203. https://doi.org/10.1073/pnas.92.6.2199

Lye, C. M., \& Sanson, B. (2011). Tension and Epithelial Morphogenesis in Drosophila Early Embryos. In M. B. T.C. T. in D. B. Labouesse (Ed.), Forces and Tension in Development (Vol. 95, pp. 145-187). Academic Press. https://doi.org/https://doi.org/10.1016/B978-0-12-385065-2.00005-0

Martin, A. C., Gelbart, M., Fernandez-Gonzalez, R., Kaschube, M., \& Wieschaus, E. F. (2010). Integration of contractile forces during tissue invagination. Journal of Cell Biology, 188(5), 735-749. https://doi.org/10.1083/jcb.200910099

Martin, A. C., Kaschube, M., \& Wieschaus, E. F. (2009). Pulsed contractions of an actin-myosin network drive apical constriction. Nature, 457(7228), 495-499. https://doi.org/10.1038/nature07522

Martinez Arias, A., \& Stewart, A. (2003). Molecular Principles of Animal Development. The Quarterly Review of Biology, 78(1), 94. https://doi.org/10.1086/377847

Maruyama, R., \& Andrew, D. J. (2012). Drosophila as a model for epithelial tube formation. Developmental Dynamics, 241(1), 119-135. https://doi.org/https://doi.org/10.1002/dvdy.22775

Mazumdar, A., \& Mazumdar, M. (2002). How one becomes many: blastoderm cellularization in Drosophila melanogaster. BioEssays, 24(11), 1012-1022. https://doi.org/https://doi.org/10.1002/bies.10184

Müller, H. A., \& Wieschaus, E. (1996). armadillo, bazooka, and stardust are critical for early stages in formation of the zonula adherens and maintenance of the polarized blastoderm epithelium in Drosophila. Journal of Cell 
Biology, 134(1), 149-163. https://doi.org/10.1083/jcb.134.1.149

Rauzi, M., Hočevar Brezavšček, A., Ziherl, P., \& Leptin, M. (2013). Physical Models of Mesoderm Invagination in Drosophila Embryo. Biophysical Journal, 105(1), 3-10. https://doi.org/https://doi.org/10.1016/j.bpj.2013.05.039

Sawyer, J. M., Harrell, J. R., Shemer, G., Sullivan-Brown, J., Roh-Johnson, M., \& Goldstein, B. (2010). Apical constriction: A cell shape change that can drive morphogenesis. Developmental Biology, 341(1), 5-19. https://doi.org/https://doi.org/10.1016/j.ydbio.2009.09.009

Sweeton, D., Parks, S., Costa, M., \& Wieschaus, E. (1991). Gastrulation in Drosophila: the formation of the ventral furrow and posterior midgut invaginations. Development, 112(3), 775 LP - 789. http://dev.biologists.org/content/112/3/775.abstract

Thomas, J. H., \& Wieschaus, E. (2004). src64 and tec29 are required for microfilament contraction during Drosophila cellularization. Development, 131(4), 863-871. https://doi.org/10.1242/dev.00989

Turner, F. R., \& Mahowald, A. P. (1977). Scanning electron microscopy of Drosophila melanogaster embryogenesis: II. Gastrulation and segmentation. Developmental Biology, 57(2), 403-416. https://doi.org/https://doi.org/10.1016/0012-1606(77)90225-1

Xue, Z., \& Sokac, A. M. (2016). -Back-to-back mechanisms drive actomyosin ring closure during Drosophila embryo cleavage. Journal of Cell Biology, 215(3), 335-344. https://doi.org/10.1083/jcb.201608025

Zalokar, M., \& Erk, I. (1976). Division and migration of nuclei during early embryogenesis of Drosophila melanogaster. J Microsc Biol Cell.

Zhang, S., \& Chen, E. H. (2008). Ultrastructural Analysis of Myoblast Fusion in Drosophila. In E. H. Chen (Ed.), Cell Fusion: Overviews and Methods (pp. 275-297). Humana Press. https://doi.org/10.1007/978-1-59745-250$2 \_16$ 

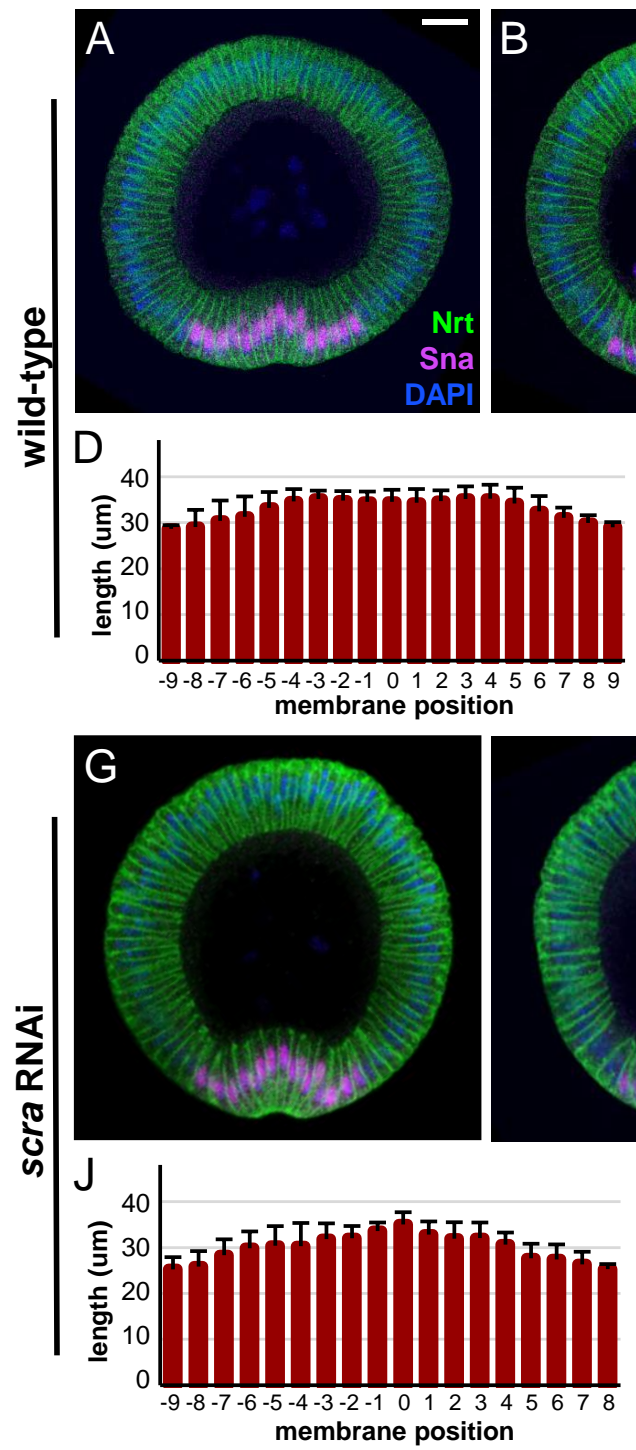

$\mathrm{E}$
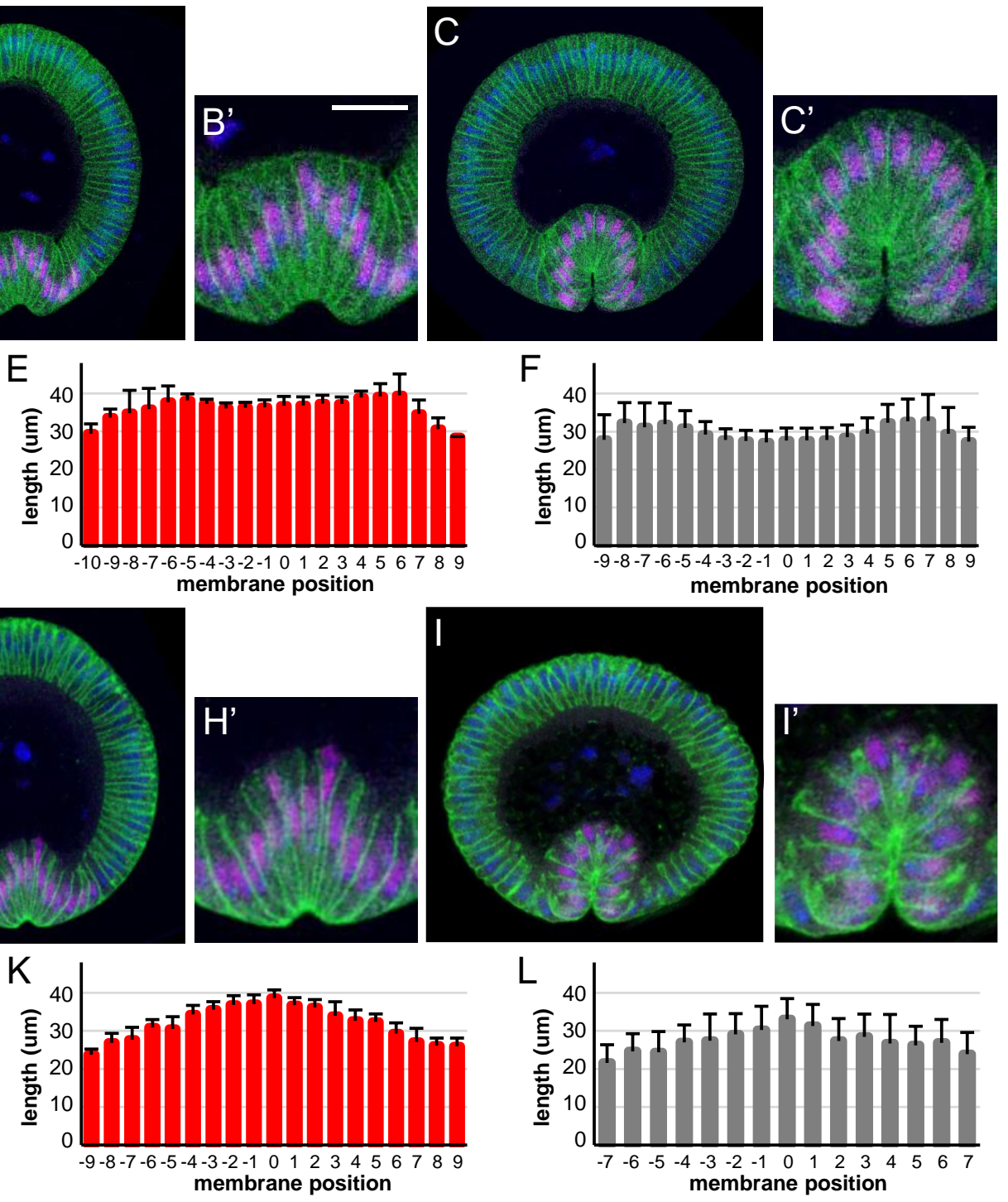

Figure 1. Basal membranes are not required for VF formation.

(A-C) Confocal immunofluorescence of heat-methanol fixed wild-type embryos during (A) apical constriction, (B) invagination, and (C) late VF formation. (D-F) Mesoderm lateral membrane lengths in wild-type embryos during (D) apical constriction ( $n=9$ embryos), (E) invagination ( $n=7$ embryos), and $(F)$ late VF formation $(n=10$ embryos). (G-I) Confocal immunofluorescence of heat/methanol-fixed scra RNAi embryos during $(G)$ apical constriction, $(H)$ invagination, and (I) late VF formation. (J-L) Mesoderm lateral membrane lengths in scra RNAi embryos during $(\mathrm{J})$ apical constriction $(\mathrm{n}=8$ embryos), (K) invagination ( $n=8$ embryos), and (L) late VF formation $\left(n=6\right.$ embryos). ( $\left.B^{\prime}\right),\left(C^{\prime}\right),\left(H^{\prime}\right)$, and (l') are $2 x$ magnifications of $(B),(C),(H)$, and (I), respectively. Images shown were cropped, rotated ventral side-down, and set against a black background. All scale bars are $20 \mu \mathrm{m}$. Data are represented as mean $\pm \mathrm{s}$. d. Membrane lengths in each of the three stages of VF formation are all significantly different across conditions (wt vs. scra RNAi, $p<0.001$ ).

See also Figures S2-S5. 
bioRxiv preprint doi: https://doi.org/10.1101/2021.04.21.440835; this version posted April 26, 2021. The copyright holder for this preprint (which was not certified by peer review) is the author/funder. All rights reserved. No reuse allowed without permission.

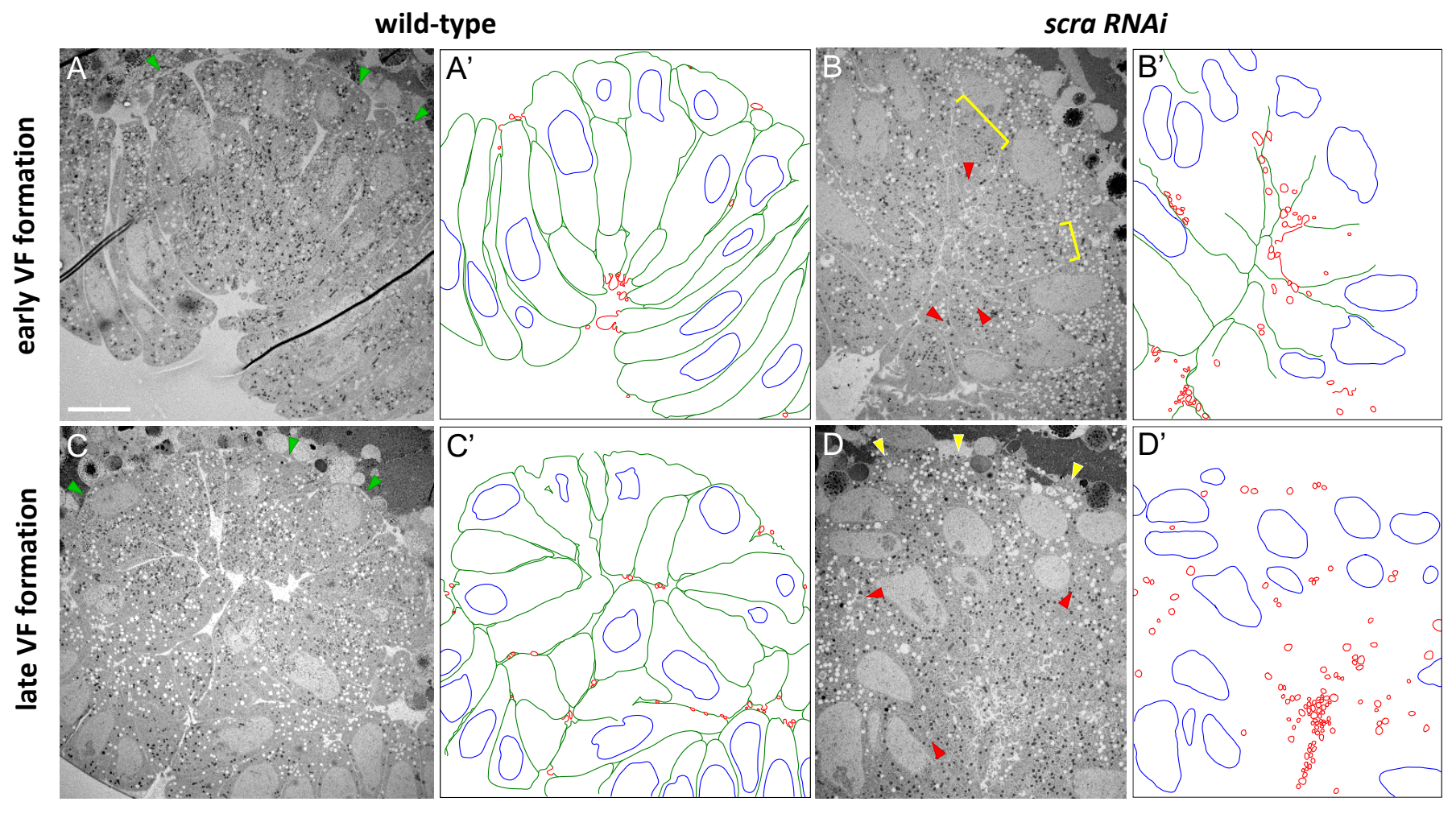

Figure 2. scra RNAi VFs remain folded despite degradation of lateral membranes

(A-D) Low mag TEM of embryo sections prepared using a combined high pressure freezing/freeze substitution method during invagination $(A, B)$ or late VF formation $(C, D)$ in wild-type $(A, C)$ and scra RNAi $(B, D)$ embryos. Interstitial spaces are visible on the basal sides of VF cells (green arrows) in wild-type embryos during both early (A) and late (B) VF formation. Basal interstitial spaces are absent (yellow brackets) and lateral membranes are starting to degrade into vesicles (red arrows) in early VF formation scra RNAi embryos (B). Basal interstitial spaces are still absent (yellow arrows) during late VF formation in scra RNAi embryos (D) and lateral interstitial spaces are no longer visible, replaced by an increased number of vesicles (red arrows). (A'-D') are hand-drawn traces of (A-D), respectively, highlighting intact interstitial spaces (green), nuclei (blue), and vesicles (red). All scale bars are $10 \mu \mathrm{m}$. 

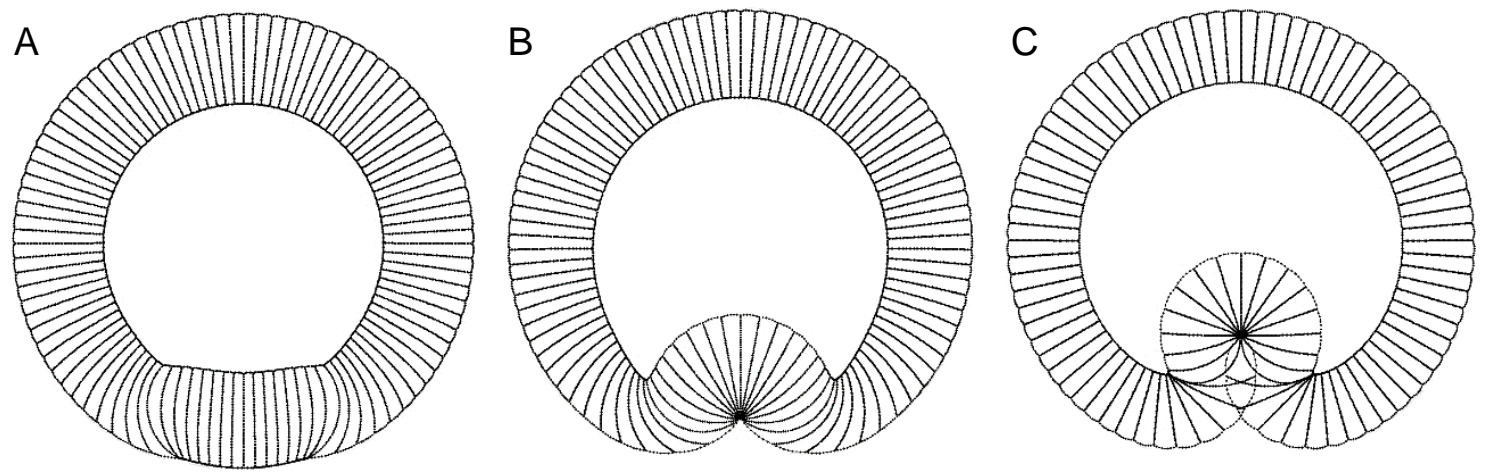

- viscosity
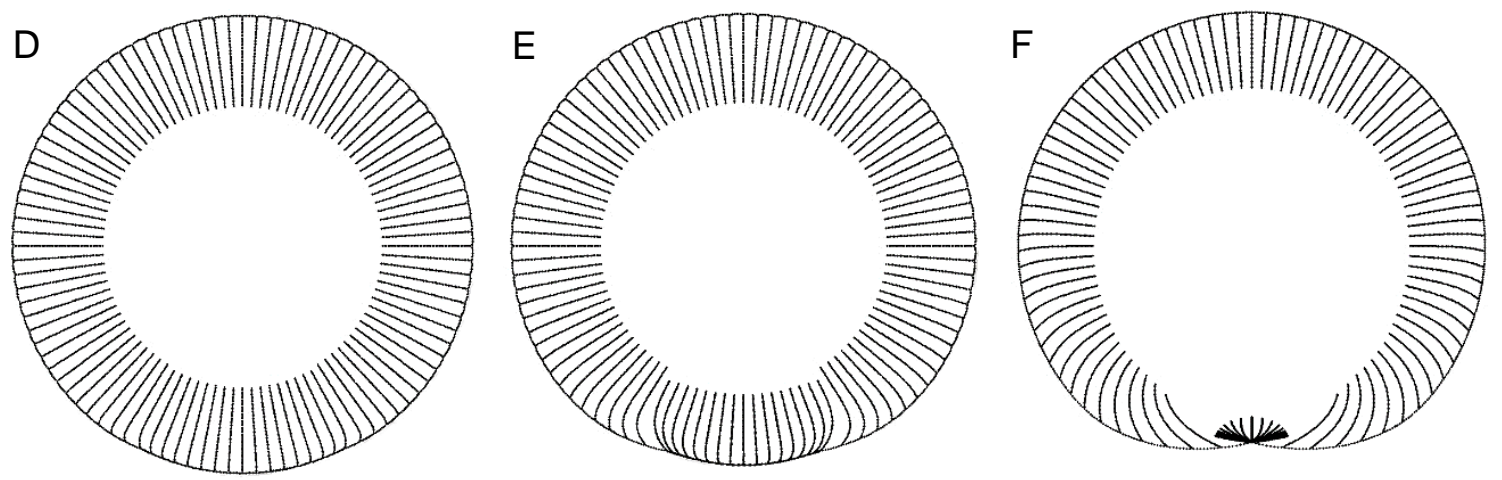

+ basal membranes
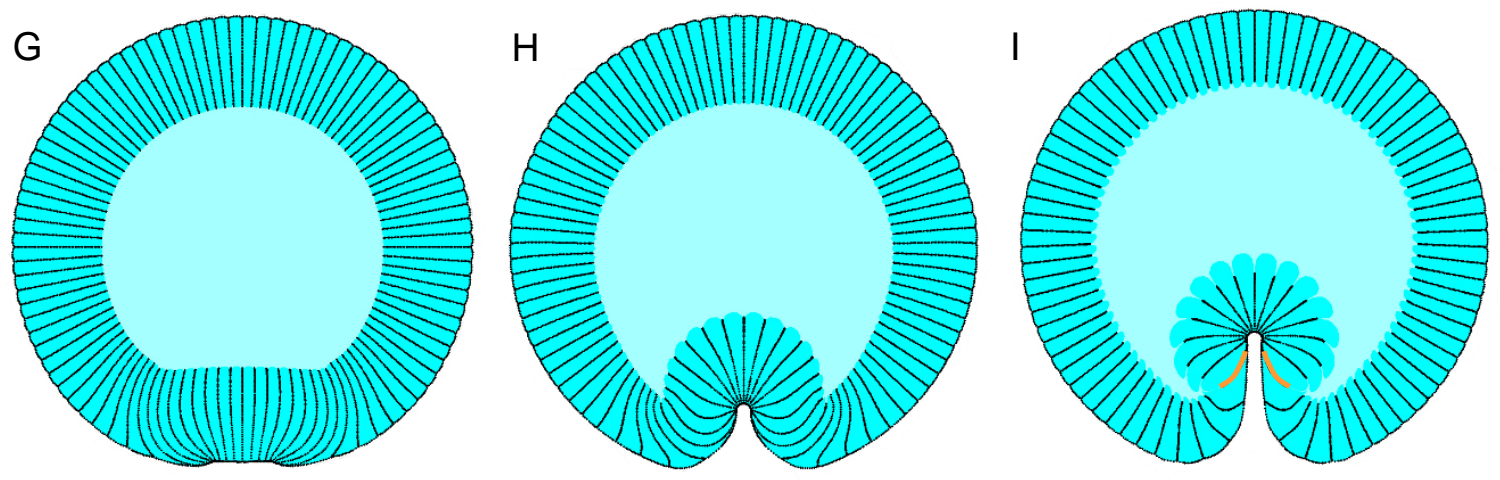

- viscosity

- basal membranes
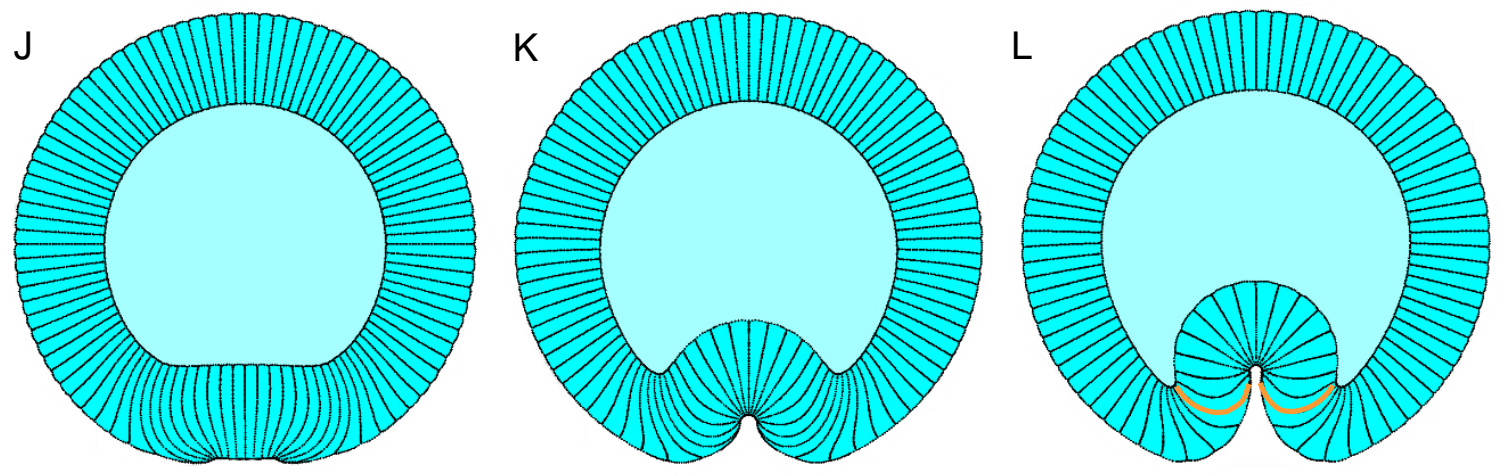

\section{scra RNAi}

+ viscosity

- basal membranes

\section{wild-type}

+ viscosity

+ basal membranes

Figure 3. VF formation without basal membranes can be explained by viscosity

Simulation results from a mathematical model of VF formation. Cells are elastic along all edges and constrained apically by modeled vitelline membrane. Simulation conditions differ as follows: (A-C) inviscid interior, basal stresses present, (D-F) inviscid interior, basal stresses absent, (G-I) viscous interior, basal stresses absent, and (J-L) viscous interior, basal stresses present. Darker blue indicates viscous material initially located inside callgie | 17 lighter blue indicates (equally) viscous material initially located inside the yolk sac. "Outer" membranes analyzed in Figure S7 are marked in orange, see also Figure S6. 


\section{SUPPLEMENTAL FIGURES}

A

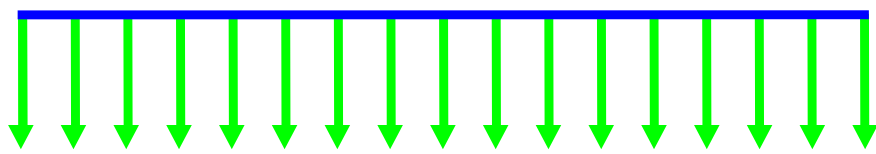

B

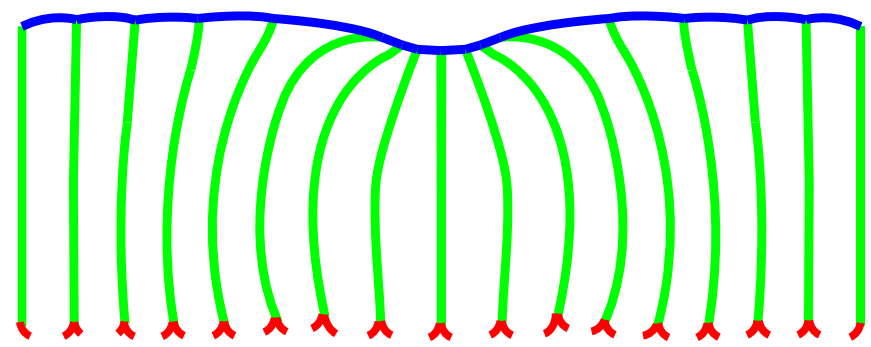

C

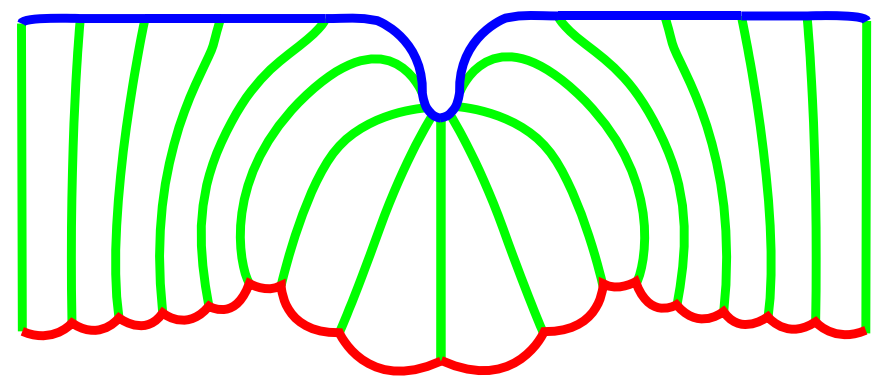

D

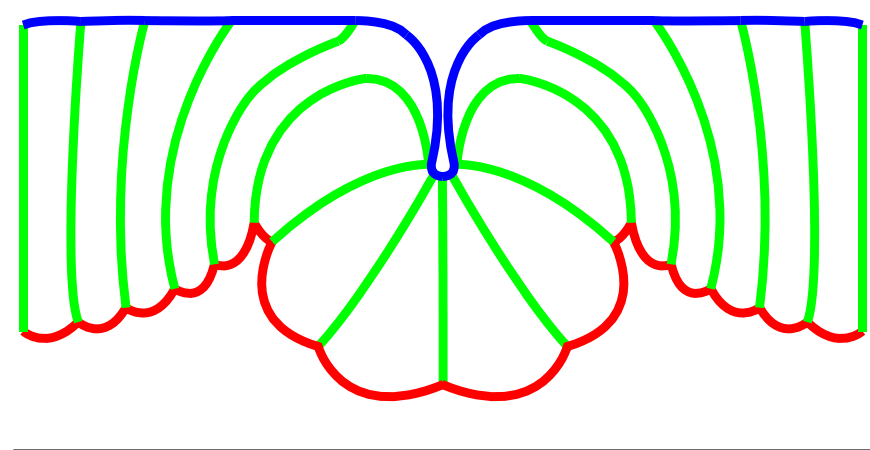

Figure S1. Schematic of membrane shape changes throughout VF formation.

(A-D) Illustration of VF formation in cross section showing shape changes in apical (blue), lateral (green), and basal (red) membranes throughout tissue folding. Lateral membranes lengthen (arrows) throughout cellularization (A). Lateral membranes of VF cells lengthen more as apical membranes constrict to initiate VF formation (B). Lateral membranes shrink back down as tissue invaginates and basal membranes form. Basal membranes seal and expand (C) as the VF continues to fold inward (D). 

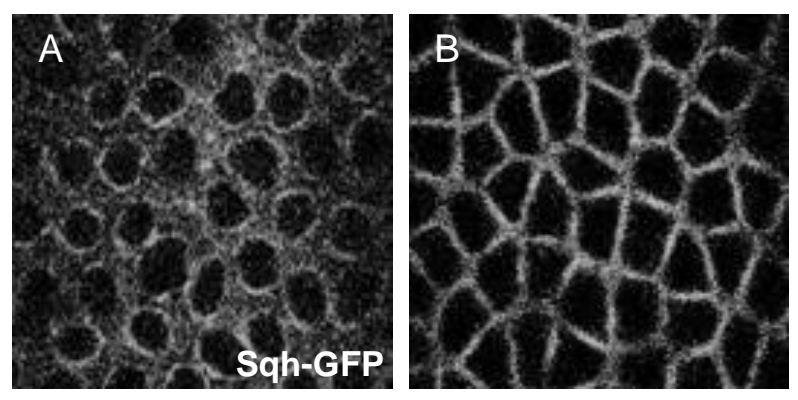

Figure S2. Acto-myosin ring closure is delayed in scra RNAi embryos.

$(A, B)$ Maximum intensity projections of the cellularizing ventral epithelium in live control $(A)$ and scra RNAi (B) embryos during late cellularization. Acto-myosin rings remain open and are misshapen in scra RNAi embryos, preventing the formation of basal membranes. 

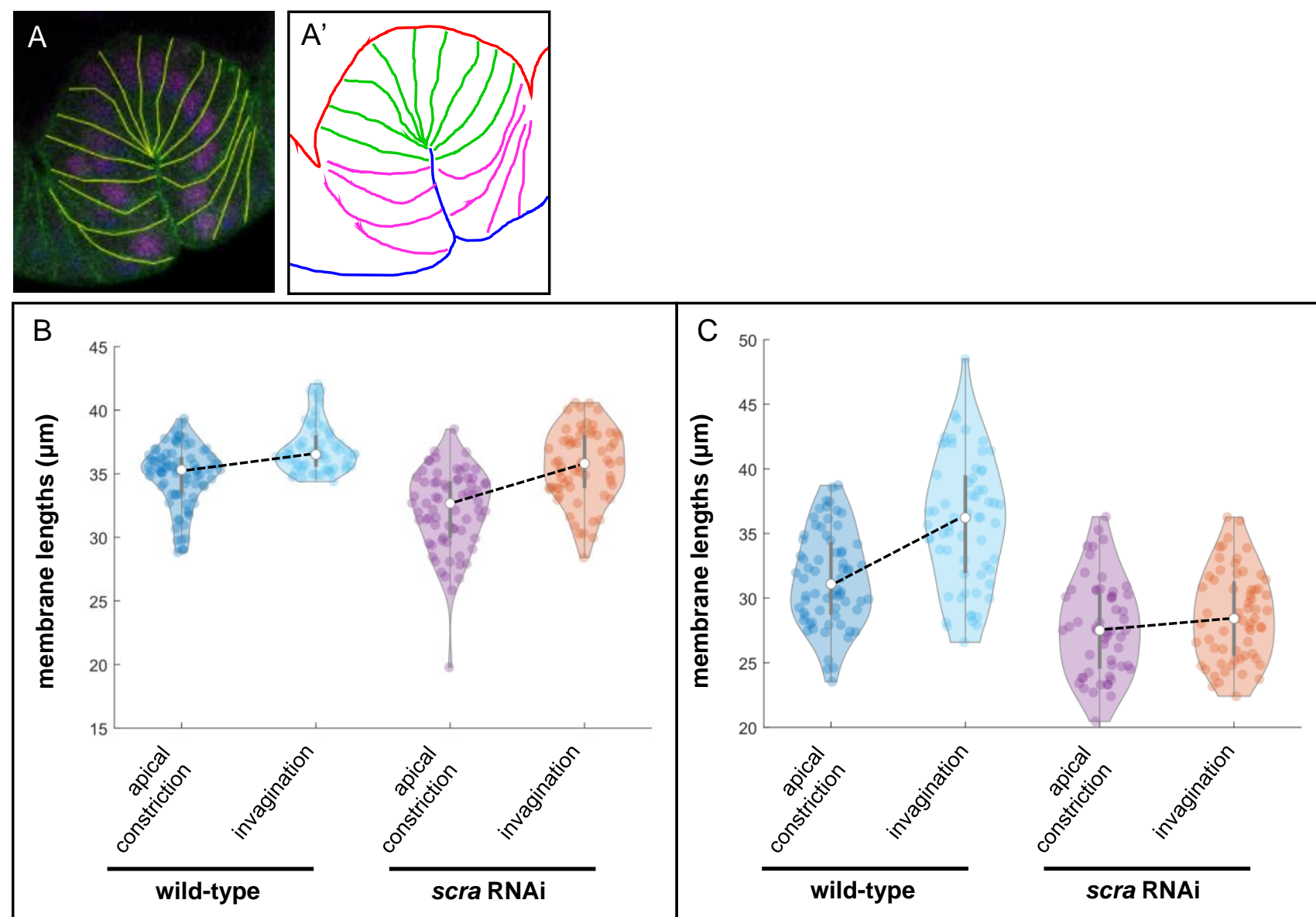

Figure S3. Central mesodermal cells lengthen more, peripheral mesodermal cells shorten less throughout VF formation in scra RNAi embryos compared to wild-type.

Example of designated central (green) and peripheral (pink) mesoderm regions shown in (A,A'). Violin plots show changes in average central $(B)$ and peripheral mesodermal membrane lengths (C) between apical constriction (ac) and invagination in wild-type ( $\mathrm{n}_{\mathrm{ac}}=9$ embryos, $\mathrm{n}_{\mathrm{invag}}=7$ embryos) and scra RNAi embryos $\left(\mathrm{n}_{\mathrm{ac}}=\mathrm{n}_{\text {invag }}=8\right.$ embryos). 


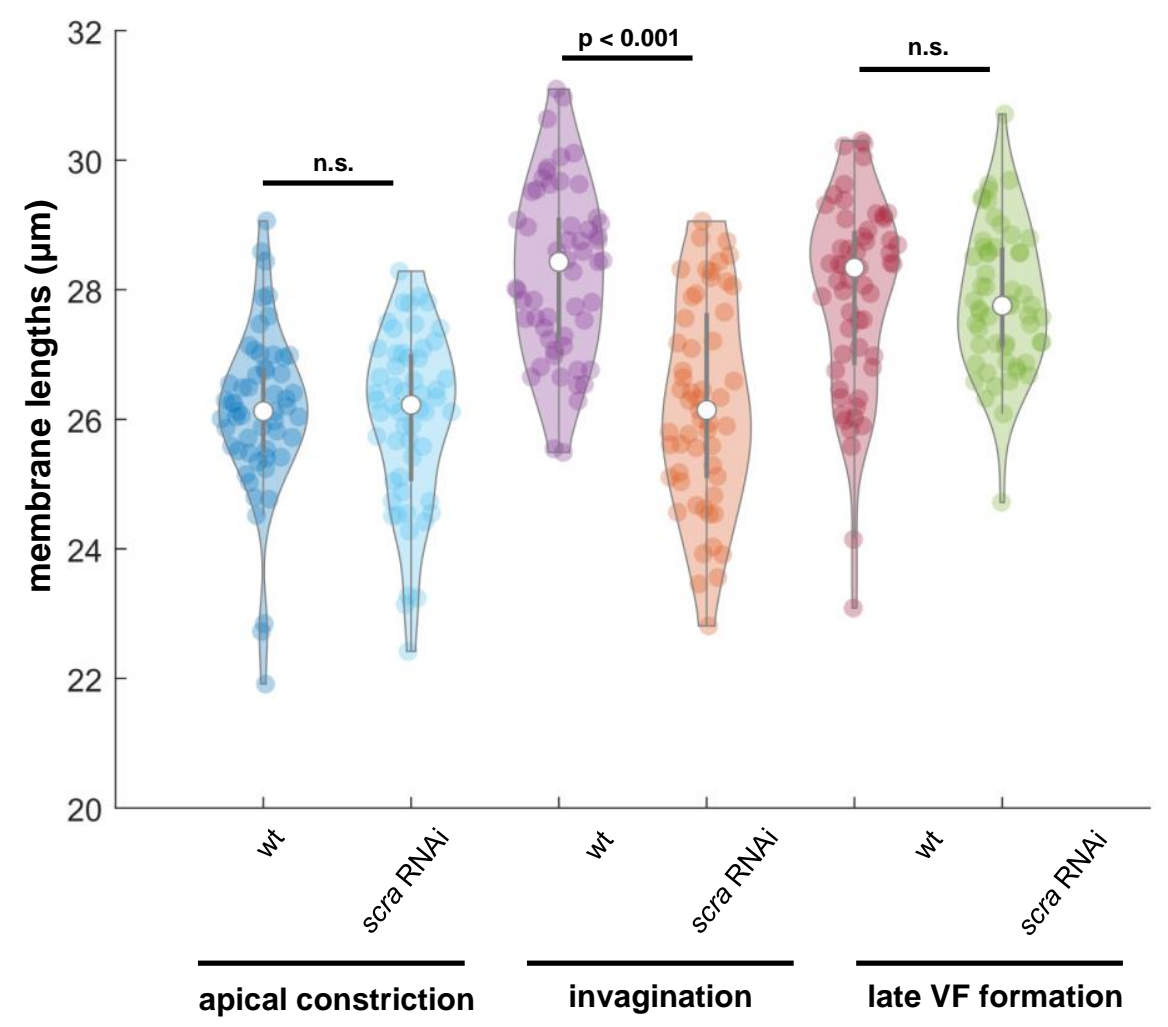

Figure S4. Non-mesodermal cells are shorter during invagination in scra RNAi embryos.

Violin plots of non-mesodermal cell lengths during apical constriction, invagination, and late VF formation in wild-type (wt) and scra RNAi embryos. Non-mesodermal cells of scra RNAi embryos do not lengthen as quickly as those in control, resulting in shorter epithelium mid VF-formation. By late VF formation, non-mesodermal cells of scra RNAi embryos have reached lengths similar to those in wild-type embryos. 
bioRxiv preprint doi: https://doi.org/10.1101/2021.04.21.440835; this version posted April 26, 2021. The copyright holder for this preprint (which was not certified by peer review) is the author/funder. All rights reserved. No reuse allowed without permission.

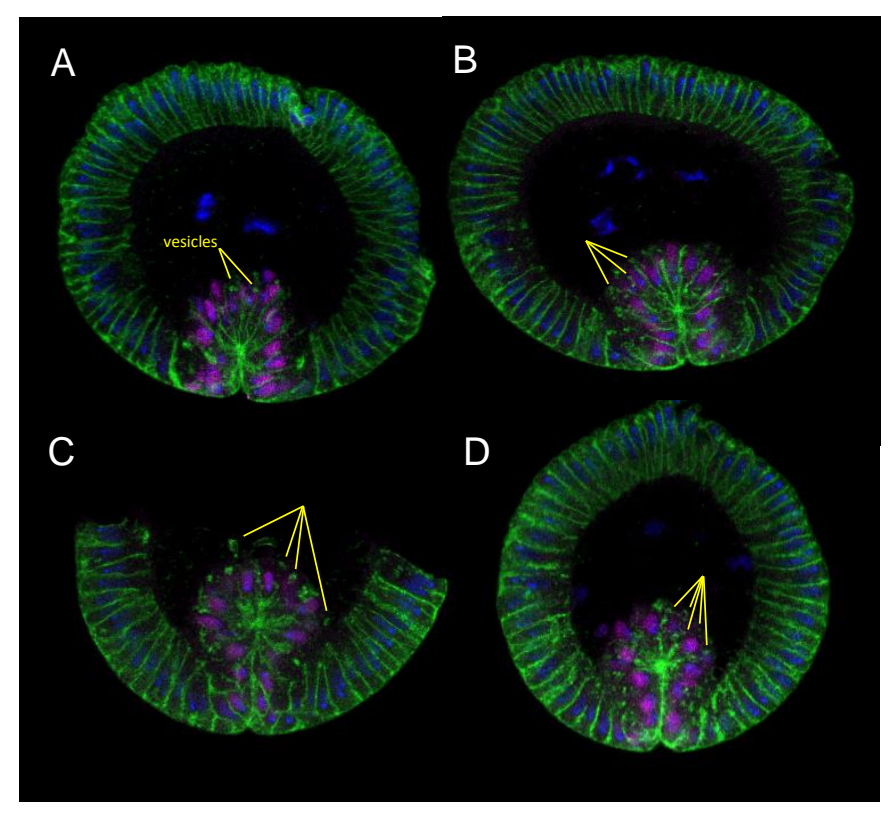

Figure S5. Membranes in scra RNAi embryos deteriorate more over time.

Confocal immunofluorescence of heat/methanol-fixed anillin RNAi embryos at increasingly older stages of gastrulation (AD). Membrane degradation increases with age, and vesicles become more numerous. Note: the embryo section in $\mathrm{C}$ is incomplete due to how it was cut, not any depleted protein expression or lack of antibody staining. 

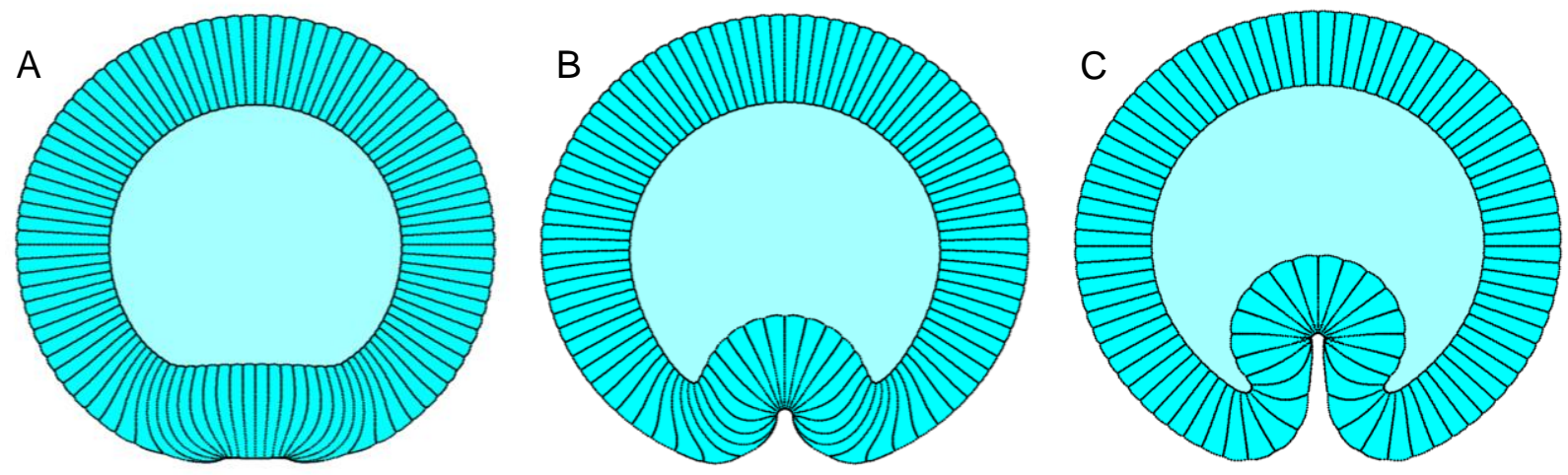

Figure S6. Model of VF formation with low basal stresses failed to replicate cell length distribution seen in vivo.

Selected simulation results at increasingly later stages (A-C) from a mathematical model of VF formation. Basal membranes are present, but active basal stresses are lower than those seen in Figures $3 \mathrm{~J}-\mathrm{L}$, resulting in inaccurate VF cell lengths. 
A

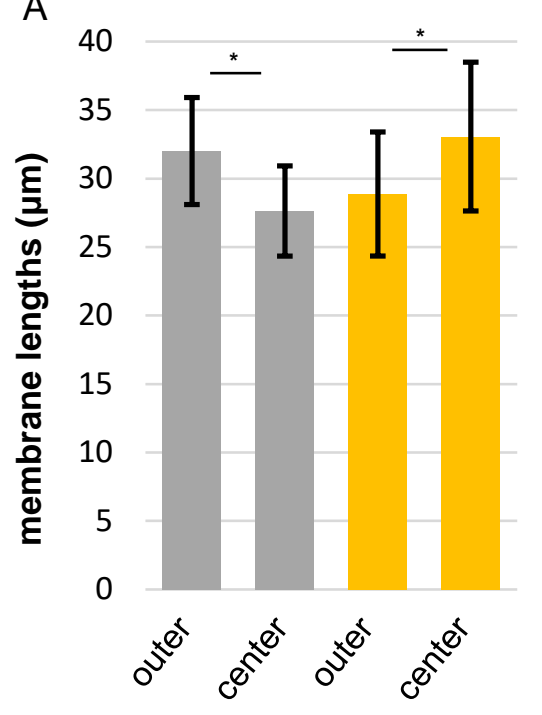

B

200

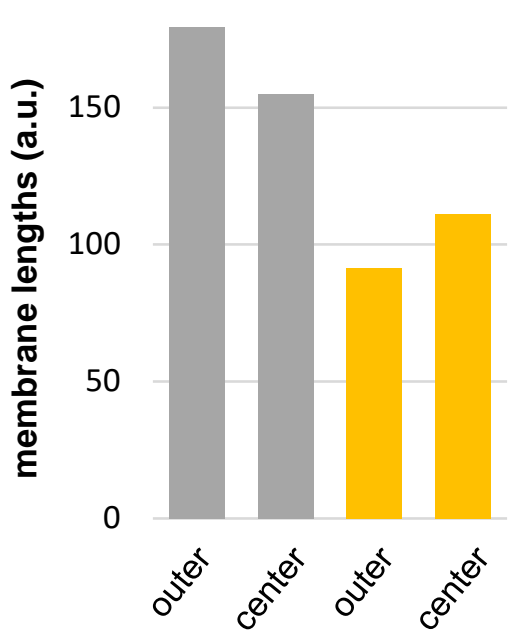

Figure S7. Model accounts for in vivo membrane length differences between membranes at edge and center of mesoderm.

(A) Lengths of membranes on outer edge of VF compared to central VF membrane in control (grey, $n=14$ embryos) and scra RNAi (yellow, $n=7$ embryos) embryos during late VF formation. Dara are represented as mean \pm s.d. Significance was determined by two-way KolmogorovSmirnov test $\left({ }^{*}=p<0.05\right)$. (B) Lengths of equivalently-located membranes in model control (grey, Figure 3I) and model scra RNAi (yellow, Figure 3L) embryos at an equivalent stage. 


\section{SUPPLEMENTAL METHODS}

Simulations were performed using the immersed boundary method [1], [2]. The basis of our simulations is a square fluid domain in which we solve for the dynamics of incompressible Stokes flow. More specifically, the governing equations read

$$
\begin{aligned}
& \tau \partial_{\mathrm{t}} u=-\nabla p+\nabla \cdot\left(\eta\left(\nabla u+\nabla u^{T}\right)\right)+f \\
& p=\gamma\left(\rho-\rho_{0}\right) \\
& \partial_{t} \rho=-\nabla \cdot(u \rho)
\end{aligned}
$$

where $u=\left(u_{x}(x, y), u_{y}(x, y)\right)$ is fluid velocity, $\rho$ is fluid density, $p$ is pressure, $\mathrm{f}$ is external force (notably that from the immersed elastic solid representing cell boundaries), $\gamma$ is (finite) fluid compressibility, $\eta(r)$ is fluid viscosity, $\rho_{0}$ is equilibrium fluid density, and $\tau$ is a small parameter with units of mass per length squared. Note, that in the limit of $\gamma \rightarrow \infty, \tau \rightarrow 0$, (1)-(3) yields incompressible Stokes flow. Note further that we allow for space dependence in the fluid viscosity $\eta$. We impose the no slip condition along the boundary of the square simulation domain.

In accordance with our recent experimental findings [3], simulated tissue is assumed to consist of cells modeled as elastic shells that enclose viscous interior. For clarity of presentation, consider first a single model cell whose equilibrium configuration is, say, a circle. We discretize its boundary into $n$ discrete nodes (at positions $r_{i}, 0 \leq i \leq n-1$ ) connected by $n$ linear springs with spring constant $E$ and rest length $L_{0}$. This amounts to assuming that cell boundaries ("membranes") are linearly elastic with a (one-dimensional) Young's modulus of $E L_{0}$. If deformed, each spring comprising the discretized boundary gives rise to a force dipole such that a discrete node $i$ is subjected to a force contribution from its adjacent spring (connecting nodes $i$ and $i+1)$ having a value of $E\left(r_{i}-L_{0}\right)\left(r_{i+1}-r_{i}\right) / r_{i}$, with $r_{i}=$ $\left\|r_{i+1}-r_{i}\right\|$ being the length of the spring joining nodes $i$ and $i+1$. If an edge is further subjected to active stress of magnitude $\mu$, there will be an additional contribution $\mu\left(r_{i+1}-r_{i}\right) / r_{i}$. Hence, force at a boundary node is given by

$$
f_{i}=E\left[\frac{\left(r_{i}-L_{0}\right)\left(r_{i+1}-r_{i}\right)}{r_{i}}+\frac{\left(r_{i-1}-L_{0}\right)\left(r_{i}-r_{i-1}\right)}{r_{i-1}}\right]+\mu\left[\frac{\left(r_{i+1}-r_{i}\right)}{r_{i}}+\frac{\left(r_{i}-r_{i-1}\right)}{r_{i-1}}\right]
$$

The two terms in each of the two square brackets correspond to force contributions from each spring adjacent to a given node. This discrete description has a continuum limit given by

$f(s, t)=\partial_{s}(T \hat{\boldsymbol{t}})$

$T=E\left(\left\|\partial_{s} r\right\|-1\right)+\mu$

$\hat{\boldsymbol{t}}=\frac{\partial_{s} r}{\left\|\partial_{s} r\right\|} \quad$,

where $r(s, t), 0 \leq s \leq L$, is the curve parametrizing the instantaneous boundary configuration, $T(s, t)$ is boundary tension, $\hat{\boldsymbol{t}}(\mathbf{s}, \mathbf{t})$ is unit tangent to the boundary, $E$ is the (1D) Young's modulus of the boundary, and $\mu(s)$ is active, "myosin-generated", stress. Definitions of $E$ and $\mu$ in (4)-(5) involve a slight obvious abuse of notation since they are not quite the same as used above Eq. (4). Note also that $\mu$ is not timedependent, whereas $E$ is constant in both time and space. Note additionally that the force term in Eq. (4) is the very same as in Eq. (1). In this way, the force density on the fluid is exactly the force density on the boundary, and hence the force balance is respected. Finally, the no slip boundary condition at the fluidsolid interface implies that a point on a cell boundary moves with the velocity of the fluid at that point: 
$\partial_{t} r(s, t)=u(r(s, t), t)=\int d r^{\prime} u\left(r^{\prime}, t\right) \delta\left(r^{\prime}-r(s, t)\right)$

with $\delta$ denoted Dirac delta-function. Taken together, Eqs. (1)-(7) specify the simulated dynamics uniquely. Our approach is exactly the same as in [2], save minor details of how Stokes equations are being integrated.

To fully describe our simulation algorithm, we need to supply a few additional details. The tissue is modeled as a set of 100 inter-connected cells arranged in a ring to represent the cross-section of an embryo. All cell boundaries are subjected to a small active "pre-stress" which amounts to a constant contribution to the $\mu$-term in Eq. (5). Cells comprising the mesoderm carry additional active stresses in their lateral and apical membranes (i.e. the corresponding $\mu$-terms have the obvious additional piecewise constant contributions). In a subset of our simulations, active stress in the basal membranes, assumed constant and present in all cells, was included as well (see main text and figure captions). The effect of the perivitelline membrane was accounted for as follows. Firstly, perivitelline space has viscosity markedly smaller than that of the cytoplasm since fluorescent beads injected into the perivitelline space diffuse considerably faster than those injected into the cells (unpublished data from our lab). To account for this, the viscosity of the fluid outside of the embryo is set to a small fraction $q=1 / 10$ of that in the interior of the embryo; this is the reason for spatial dependence of $\eta$ in Eq. (1). Additionally, we include a soft repulsive potential between the cells and the model vitelline membrane, represented by a circle with a diameter slightly larger than the initial radius of the model embryonic surface. The repulsive potential is set large enough to ensure that all nodes parameterizing cell boundaries always remain within the model vitelline membrane. Note that in the incompressible limit the volume of the perivitelline space (i.e. the space between the surface of the embryo and the vitelline membrane) remains constant. In this way, in the limit of small $q$, the dynamics amounts to neglecting the viscosity of the perivitelline fluid. This is justified by the following argument. Assuming viscosity of the perivitelline fluid is similar to that of water, it is 1000-fold less viscous than the cytoplasm [3]. Typical width of the perivitelline space is 4 microns (unpublished data from our lab), whereas the diameter of the embryo is 170 microns. Thus, the ratio of contributions to viscous dissipation from the cytoplasm and the perivitelline space is expected to be $(1000 / 1)^{*}(4 / 180) \sim 20$. Hence, the contribution of the perivitelline space is only $\sim 5 \%$ and can therefore be neglected. Finally, the implementation of the immersed boundary is adopted from [2] without modification. In particular, we use the discrete approximation of delta-peak given by Equation (25) in [2]. A major drawback of the immersed boundary method is poor volume conservation. This is exhaustively addressed in [2] by employing a correction based on Chorin projection. Our implementation uses this exact method without any modifications. Note that this correction is exact in the sense that it involves neither small parameters nor artificial "fudge-terms". Finally, the immersed boundary method has one additional source of error in volume conservation that it shares with any other off-grid approach: even if the immersed solid boundary is advected by incompressible flow, the enclosed volume will drift if fluid escapes between discrete nodes. This is very easy to control provided the density of nodes discretizing the surface is chosen sufficiently high. However, in our simulations, some membranes stretch considerably such that the density of solid nodes falls well below that of the fluid nodes. To prevent this, we refine the discretization of cell boundaries dynamically. Specifically, if in a given simulation time-step the length of an edge exceeds twice its original length, it is subdivided into two distinct edges. To ensure that the new composite edge has the same material properties as the old edge, the rest lengths of the newly introduced edges are re-scaled by $1 / 2$, whereas their spring-constants are doubled. In this way, the density of nodes discretizing the immersed solid is maintained constant such that accurate volume conservation is imposed. 
In summary, the algorithm employed in our simulations is as follows:

Initialize boundary configuration and all dynamic fields.

Loop steps 1-8.

Step 1. Using instantaneous configuration of the tissue, compute the spatial profile of viscosity $\eta$ (set to be smaller outside of the embryo than inside by a factor of $q$ ).

Step 2. Using instantaneous configuration of the tissue, compute total force density at the boundary involving three contributions: (1) elastic stress, (2) active stress, and (3) confining force from the vitelline membrane.

Step 3. Spreading step of the immersed boundary method:. Force density on the solid is "spread" to the fluid using the discrete delta-peak approximation. For details, refer e.g. to [2].

Step 4. Evolve fluid velocity according to Eqs. (1)-(3).

Step 5. Correct for volume conservation in the flow computed in the previous step using Chorin projection (implemented with the help of Fast Fourier Transform).

Step 6. Interpolation step of the immersed boundary method. Velocity from the fluid grid is interpolated to the solid nodes.

Step 7. Solid nodes are evolved through a time-step using node velocities from Step 6 (using the Euler forward explicit scheme).

Step 8. If a solid edge is stretched more than twice its original length, it is split in two and its properties are re-scaled accordingly (see above).

Note that our strategy is almost exactly the same as that in [2], save minor details of how Stokes equations are integrated and the dynamical re-meshing employed to maintain constant (surface) density of solid nodes.

All of our code is thoroughly annotated and is available online under our lab github repository: ??

\section{SUPPLEMENTAL REFERENCES}

[1] C. S. Peskin, "Flow patterns around heart valves: A numerical method," J. Comput. Phys., vol. 10, no. 2, pp. 252-271, 1972, doi: https://doi.org/10.1016/0021-9991(72)90065-4.

[2] C. S. Peskin and B. F. Printz, "Improved Volume Conservation in the Computation of Flows with Immersed Elastic Boundaries," J. Comput. Phys., vol. 105, no. 1, pp. 33-46, 1993, doi: https://doi.org/10.1006/jcph.1993.1051.

[3] K. Doubrovinski, M. Swan, O. Polyakov, and E. F. Wieschaus, "Measurement of cortical elasticity in Drosophila melanogaster embryos using ferrofluids," Proc. Natl. Acad. Sci., vol. 114, no. 5, pp. 1051-1056, Jan. 2017, doi: 10.1073/pnas.1616659114. 